\title{
BLOWUP ESTIMATES FOR A FAMILY OF SEMILINEAR SPDES WITH TIME-DEPENDENT COEFFICIENTS
}

\section{Abner Alvarez, José Alfredo López-Mimbela And Nicolas Privault}

Abstract. We investigate the blowup and stability of semilinear stochastic partial differential equations with time-dependent coefficients using stopping times of exponential functionals of Brownian martingales and a non-homogeneous heat semigroup. In particular we derive lower bounds for the probability of blowup in finite time, and we provide sufficient conditions for the existence of global positive solutions.

Mathematics subject classification (2010): 60H15, 35R60, 35K58, 35B40, 35B44.

Keywords and phrases: stochastic partial differential equations, blowup of semilinear equations, weak and mild solutions, exponential functionals of Brownian motion.

\section{REFERENCES}

[1] M. Abramowitz and I.A. Stegun, Handbook of mathematical functions with formulas, graphs, and mathematical tables, volume 55. Dover Publications, New York, 1972. 9th Edition.

[2] V.V. Baklan, On the existence of solutions of stochastic equations in Hilbert space. Dopovidi Akad. Nauk Ukraïn. RSR., 1963:1299-1303, 1963.

[3] E.B. Davies, Heat kernels and spectral theory, volume 92 of Cambridge Tracts in Mathematics. Cambridge University Press, Cambridge, 1989.

[4] M. Dozzi, E.T. Kolkovska, and J.A. López-Mimbela, Exponential functionals of Brownian motion and explosion times of a system of semilinear SPDEs. Stochastic Anal. Appl., 31(6):975-991, 2013.

[5] M. Dozzi and J.A. López-Mimbela, Finite-time blowup and existence of global positive solutions of a semi-linear SPDE. Stochastic Process. Appl., 120(6):767-776, 2010.

[6] H. Fujita, On some nonexistence and nonuniqueness theorems for nonlinear parabolic equations. In Nonlinear Functional Analysis (Chicago, Ill., 1968), volume XVIII, Part 1 of Proc. Sympos. Pure Math., pages 105-113, Providence, RI, 1970. Amer. Math. Soc.

[7] I. Karatzas and S. Shreve, Brownian motion and stochastic calculus, volume 113 of Graduate Texts in Mathematics. Springer-Verlag, New York, second edition, 1991.

[8] N.V. Krylov and B.L. Rozovskii, Stochastic evolution equations. Journal of Soviet Mathematics, 14:1233-1277, 1981.

[9] J.A. López-Mimbela and A. Pérez, Finite time blow up and stability of a semilinear equation with a time dependent Lévy generator. Stoch. Models, 22(4):735-752, 2006.

[10] J.A. López-Mimbela and A. Pérez, Global and nonglobal solutions of a system of nonautonomous semilinear equations with ultracontractive Lévy generators. J. Math. Anal. Appl., 423:720-733, 2015.

[11] G. Lv and J. Duan, Impacts of noise on a class of partial differential equations. J. Differential Equations, 258(6):2196-2220, 2015.

[12] E.M. Ouhabaz and F.Y. Wang, Sharp estimates for intrinsic ultracontractivity on $C^{1, \alpha}$-domains. Manuscripta Math, 122(2):229-244, 2007.

[13] É. Pardoux, Équations aux dérivées partielles stochastiques nonlinéares monotones: étude de solutions fortes de type Itô. PhD thesis, Université Paris-Orsay, 1975.

[14] N.H. Pavel, Nonlinear evolution operators and semigroups. Applications to partial differential equations, volume 1260 of Lecture Notes in Math. Springer-Verlag, Berlin, 1987.

[15] A. Pazy, Semigroups of linear operators and applications to partial differential equations, volume 44 of Applied Mathematical Sciences. Springer-Verlag, New York, 1983. 
[16] M. Sanz-Solé and P.-A. Vuillermot, Equivalence and Hölder-Sobolev regularity of solutions for a class of non-autonomous stochastic partial differential equations. Ann. Inst. H. Poincaré Probab. Statist., 39(4):703-742, 2003.

[17] J. Seidler, Da prato-Zabczyk's maximal inequality revisited. Math. Bohem., 118(1):67-106, 1993.

[18] G. Teschl, Ordinary Differential Equations and Dynamical Systems, volume 40 of Graduate Studies in Mathematics. American Mathematical Society, Providence, RI, 2012.

[19] M. Veraar, Non-autonomous stochastic evolution equations and applications to stochastic partial differential equations. J. Evol. Equ., 10(1):85-127, 2010.

[20] M. Veraar and J. Zimmerschied, Non-autonomous stochastic Cauchy problems in Banach spaces. Studia. Math., 185:1-34, 2008.

[21] M. Yor, Exponential functionals of Brownian motion and related processes. Springer Finance. Springer-Verlag, Berlin, 2001. 